\title{
Next-generation In Silico Cardiac Electrophysiology Through Immersed Grid Meshfree Modeling: Application To Simulation Of Myocardial Infarction
}

\author{
Konstantinos A Mountris ${ }^{1,2}$, Esther Pueyo ${ }^{1,2}$ \\ ${ }^{1}$ Aragon Institute of Engineering Research, University of Zaragoza, IIS Aragon, Zaragoza, Spain \\ ${ }^{2}$ CIBER in Bioengineering, Biomaterials \& Nanomedicine (CIBER-BBN), Spain
}

\begin{abstract}
State-of-art simulators of cardiac tissue electrophysiology are commonly based on the Finite Element Method (FEM). FEM is known to be a robust and accurate numerical method, but its accuracy highly depends on the quality of the mesh. Generating a good-quality mesh may be cumbersome and time consuming for models with complex geometries, such as those representing the anatomy of human organs. This limitation restricts the clinical application of FEM. To overcome this challenge, we propose the use of a meshfree method, the Moving Kriging Mixed Collocation (MKMC) method for in silico cardiac electrophysiology. MKMC is a purely meshfree method requiring the definition of a point cloud rather than a mesh. We propose the construction of the point cloud as an immersed grid of points generated automatically from image data. In simulations on a swine biventricular model, both under baseline and myocardial infarction conditions, we demonstrate the capability of the MKMC method to generate results in very good agreement with FEM while alleviating the mesh requirement. Differences in local activation time and action potential duration between MKMC and FEM are in mean below 3\%. The proposed MKMC method represents a promising alternative to FEM for cardiac in silico investigations with the potential to be integrated in the clinic.
\end{abstract}

\section{Introduction}

Myocardial infarction (MI) due to ischemic heart disease is acknowledged as the most common mortality cause worldwide [1]. Following MI, the heart undergoes electrophysiological and structural remodeling, including scar formation at the infarct area and accumulation of fibrosis at the surrounding border zone. MI-induced remodeling provides the substrate for the initiation and maintenance of ventricular arrhythmias. Depending on the extent of remodeling, arrhythmias following MI can be lethal [2].

Computational modeling and simulation has proved to be a valuable resource for the understanding, prediction and treatment of MI-related ventricular arrhythmias [3]. Most state-of-art cardiac electrophysiology simulators use the Finite Element Method (FEM) to solve the bidomain model or its simplified version, known as the monodomain model. While FEM is a very robust numerical method, its application in the clinical setting remains limited. This can be to a good extent explained by the requirement for a high-quality mesh to ensure accurate simulation results. Due to the complexity of the cardiac anatomy, mesh generation may be cumbersome and require engineering expertise that is not expected to be available in a clinical setting.

To overcome mesh-related limitations, alternative meshfree methods, such as the Smooth Particle Hydrodynamics (SPH) [4] and the Mixed Collocation (MC) methods [5,6], have been proposed for in silico cardiac electrophysiology. In SPH and MC, the mesh requirement is alleviated and a discretization of the cardiac anatomy in arbitrarily distributed points is sufficient to obtain accurate simulation results. This property renders these methods as promising alternatives to FEM, especially for clinical applications. Furthermore, MC presents higher flexibility since different trial functions (e.g. moving least squares, radial basis functions) may be used to construct the meshfree approximation. For each point, the meshfree approximation is constructed on a group of neighbor points (support domain). Theoretically, support domains with different shape (cubic, spherical, etc.) may be used. In practice, badly-shaped support domains may lead to accuracy deterioration.

In this work, we propose a novel approach to solve the monodomain model using the Moving Kriging interpolation [7] as the trial function in the MC method and the notion of the immersed grid to generate the cardiac anatomy discretization. In contrast to FEM meshes, immersed grids are easily generated by embedding a regular grid of points inside a triangular surface mesh of the anatomy. Moreover, the regularity of the points distribution always leads to well-shaped support domains that ensure the accuracy of the meshfree approximation. To evaluate our method's performance, we simulate electrical propagation in a swine biventricular model with and without a myocardial infarction scar in the left ventricle. We compare the MC solution on the immersed grid with the solution of a FEM simula- 
tion on a tetrahedral mesh representation of the biventricular model. We show very good agreement between the two solutions. The advantage of the proposed method over FEM is that, due to the immersed grid approach, accurate meshfree models can be generated automatically, rendering this technique suitable for use in the clinical setting.

\section{Materials and methods}

\subsection{Moving Kriging Mixed Collocation}

The monodomain model is used to describe the electrical activity of the cardiac tissue:

$$
\begin{array}{ll}
\partial V / \partial t=-I_{\text {ion }}(V) / C+\nabla \cdot(\boldsymbol{D} \nabla V) & \text { in } \Omega \\
\boldsymbol{n} \cdot(\boldsymbol{D} \nabla V)=0 & \text { in } \partial \Omega
\end{array}
$$

where $\partial V / \partial t$ is the time derivative of the transmembrane voltage, $I_{i o n}$ is the total ionic current, $C$ is the cell capacitance per unit surface area and $D$ is the diffusion tensor. $\Omega$ and $\partial \Omega$ denote the domain of interest and its boundary, and $\boldsymbol{n}$ is the outward unit vector normal to the boundary.

The formulation of the Moving Kriging Mixed Collocation (MKMC) method is derived from the Petrov-Galerkin weak form of the monodomain model. Using the operator splitting method [8] with the Dirac function and Moving Kriging interpolation [7] as test and trial functions, the equations are given by:

$$
\begin{array}{ll}
\partial V_{I} / \partial t=-I_{\text {ion }}\left(V_{I}\right) / C & \text { in } \Omega \\
\sum_{i=1}^{m} \phi_{I}^{i} \partial V_{i} / \partial t=\sum_{i=1}^{m} \nabla \cdot \boldsymbol{D} \nabla^{T} \phi_{I}^{i} V_{i} & \text { in } \Omega \\
\sum_{i=1}^{m} \boldsymbol{n} \cdot \boldsymbol{D} \boldsymbol{\nabla} \phi_{I}^{i} V_{i}=0 & \text { in } \partial \Omega
\end{array}
$$

where $I=1,2, \ldots, N$ is the $I^{t h}$ point of the $N$ points discretizing $\Omega, i=1,2, \ldots, m$ is the $i^{t h}$ point of the $m$ points in the support domain of $I$ (being $I$ one of these points) and $\phi_{I}^{i}$ is the value of the Moving Kriging interpolation vector $\phi_{I}$ for component $i$. $\phi_{I}$ is given by:

$$
\phi_{I}=p_{I} \boldsymbol{A}+\boldsymbol{c}_{I} \boldsymbol{B}
$$

where $\boldsymbol{p}_{I}$ is the polynomial basis vector with rank $k$ and $c_{I}$ is the correlation function vector. In this work, we use the quadratic polynomial basis:

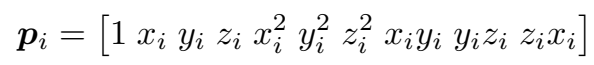

where $x_{i}, y_{i}, z_{i}$ are spatial coordinates. We use the Gaussian correlation function to obtain:

$$
\begin{aligned}
\boldsymbol{c}_{i} & =\left[\begin{array}{llll}
\gamma_{i 1} & \gamma_{i 2} & \ldots & \gamma_{i m}
\end{array}\right] \\
\gamma_{i j} & =e^{-\theta r_{i j}^{2}}
\end{aligned}
$$

where $r_{i j}$ is the Euclidean distance between points $i$ and $j$ $(i, j=1,2, \ldots, \mathrm{m})$, and $\theta=1.05$ is the shape parameter.
The matrices $\boldsymbol{A}$ and $\boldsymbol{B}$ are obtained by:

$$
\begin{aligned}
& \boldsymbol{A}=\left(\boldsymbol{P}^{T} \boldsymbol{C}^{-1} \boldsymbol{P}\right)^{-1} \boldsymbol{P}^{T} \boldsymbol{C}^{-1} \\
& \boldsymbol{B}=\boldsymbol{C}^{-1}(\boldsymbol{I}-\boldsymbol{P} \boldsymbol{A})
\end{aligned}
$$

where $\boldsymbol{I}$ is the $m \times m$ identity matrix, $\boldsymbol{P}$ is the $m \times k$ polynomial basis matrix with rows $\boldsymbol{p}_{i}$ and $\boldsymbol{C}$ is the $m \times$ $m$ correlation matrix for the $m$ points in the $I^{t h}$ point's support domain, with rows $\boldsymbol{r}_{i}, i=1,2, \ldots, m$.

\subsection{Swine biventricular model}

The immersed grid discretization for the MKMC method can be obtained directly from each individual's segmentation image. The segmentation image voxels inside the model domain $\Omega$ are converted to a regular grid of $3 \mathrm{D}$ points. Points on the domain boundary $\partial \Omega$ are obtained generating a smooth triangular surface mesh of the segmentation. Alternatively, a regular grid of points is generated inside the bounding box (BB) of the surface mesh of $\partial \Omega$. Subsequently, an inclusion test algorithm is applied to discard any point $p \notin \Omega$ (Figure 1 ). In this way, mapping complex anatomical structures from the segmentation data to the model's point discretization is straightforward.
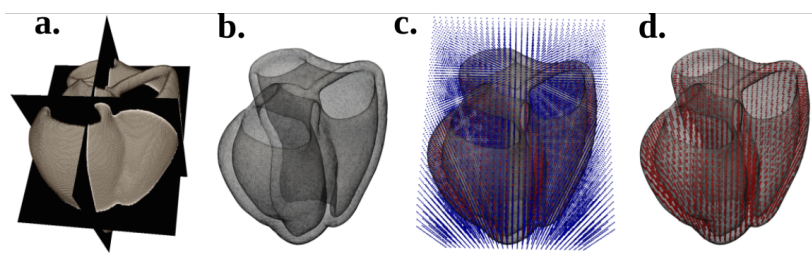

Figure 1. Immersed grid generation. a) Image segmentation; b) surface mesh extraction; c) points generation in bounding box; d) inclusion test algorithm application.

We considered a swine biventricular model with a myocardial infarction scar in the left ventricle provided to us from a previous study [9]. The biventricular model was made available in the form of a tetrahedral mesh. To generate the immersed grid, we extracted the surface of the tetrahedral mesh and constructed the grid of points in the bounding box of the surface mesh as in Figure 1. The mesh partitions (cardiac tissue, connective tissue, scar tissue) were mapped to the grid points during the inclusion algorithm application. No border zone partition was available in the mesh, so it was not considered in the immersed grid. We divided the cardiac tissue into endocardium, midmyocardium, and epicardium compartments with ratio 5:2:3 based on the transmurality data of the mesh.

Myocardial fiber direction at the mesh vertices was generated using a rule-based method [10]. Moving Kriging interpolation was used to interpolate the fiber direction vectors from the vertices to the grid points (Figure 2). 

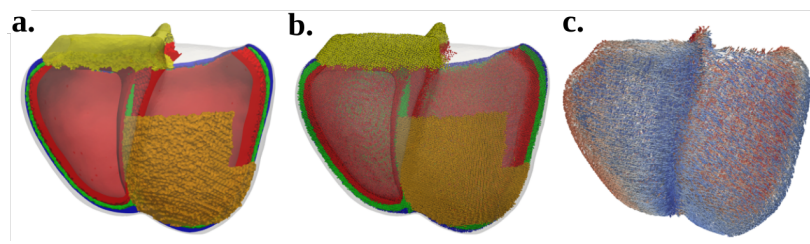

Figure 2. a) and b) Mesh and grid endocardium (red), midmyocardium (green), epicardium (blue), scar (orange) and connective (yellow) tissue; c) Immersed grid fiber vectors.

\subsection{Simulation configuration}

The OH́ara-Virág-Varró-Rudy ventricular cell model was used to represent human ventricular cell electrophysiology. Action potential (AP) propagation in the tissue was simulated using the MKMC method in two different conditions: baseline (i.e. no scar in the biventricular model) and myocardial infarction (i.e. with the scar included in the model). The results obtained using the MKMC method were compared with those obtained from a FEM simulation. The value for the longitudinal conductivity was $\sigma_{t}=2.8 \mathrm{mS} / \mathrm{cm}$ for the cardiac tissue and $\sigma_{s}=0$ $\mathrm{mS} / \mathrm{cm}$ for the scar and connective tissue. The transversalto-longitudinal conductivity ratio was set $\rho=0.25$.

To apply realistic stimulation on the biventricular model, a Purkinje network on the endocardial surfaces of the two ventricles was generated using a fractal-tree generation algorithm [11]. Purkinje-Myocardial Junctions (PMJs) were computed applying a range query algorithm with spherical search area of radius $R=1.5 \mathrm{~mm}$, centered at each end-node of the Purkinje network. We did not implement AP propagation in the Purkinje network. Instead, periodical stimuli of 1-ms duration at a cycle length of $1 \mathrm{~s}$ were directly applied onto the PMJs. The amplitude was double the diastolic threshold. To ensure realistic activation times matching published data [12], we partitioned the PMJs into four groups. PMJs at the left ventricle apex were stimulated at time $0 \mathrm{~ms}$, whereas a delay of 7, 4 and $11 \mathrm{~ms}$ was applied at PMJs located at the left ventricle base, the right ventricle apex and the right ventricle base, respectively.

Simulation results from the MKMC method were compared with those from a FEM simulation. The comparison was made in terms of the local activation time (LAT) and the AP duration at $90 \%$ repolarization $\left(\mathrm{APD}_{90}\right)$ assessing the effects of myocardial infarction on LAT and $\mathrm{APD}_{90}$.

\section{Results}

MKMC and FEM simulation results were in good agreement, as illustrated in Figures 3 and 4. The mean relative difference for the whole ventricles' LAT was $1.6 \%$ at baseline (mean LAT: $24.3 \mathrm{~ms}$ for MKMC vs $23.9 \mathrm{~ms}$ for FEM) and $2.9 \%$ for myocardial infarction conditions (mean LAT:
$26.7 \mathrm{~ms}$ for MKMC vs $25.9 \mathrm{~ms}$ for FEM). The mean relative difference for $\mathrm{APD}_{90}$ was $2.9 \%$ at baseline (MKMC: $280.5 \mathrm{~ms}$, FEM: $272.1 \mathrm{~ms}$ ) and $2.6 \%$ for myocardial infarction conditions (MKMC: $277.3 \mathrm{~ms}$, FEM: $269.9 \mathrm{~ms}$ ).

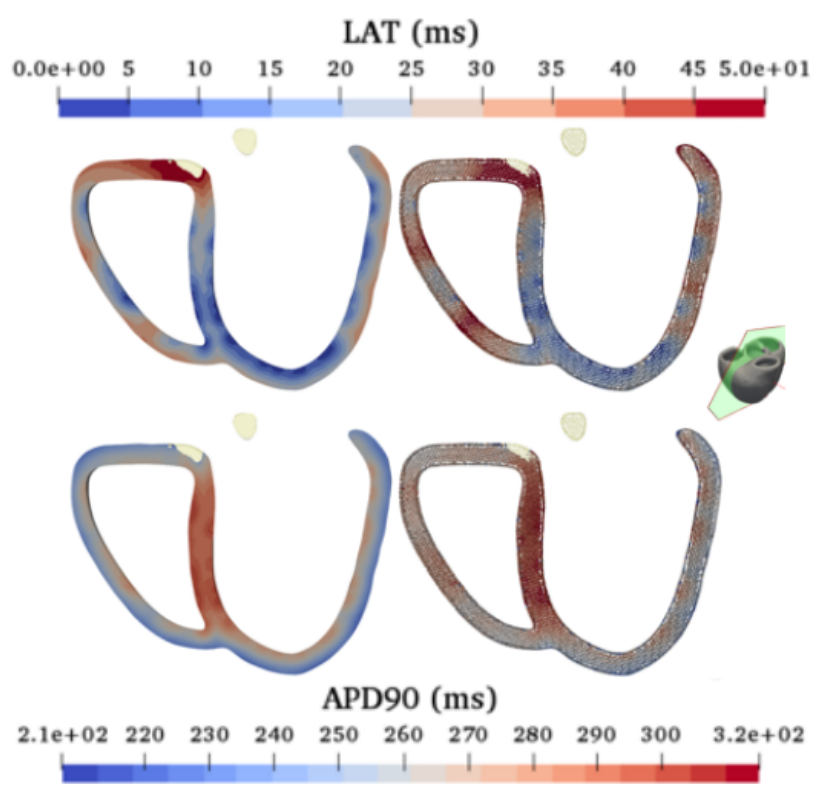

Figure 3. Local activation time (top) and AP duration at 90\% repolarization (bottom) for FEM (left) and MKMC (right) simulations at baseline conditions.

The above results demonstrate similar effects for the infarction simulation as quantified from MKMC and FEM simulations. Mean LAT increased by $2.4 \mathrm{~ms}$ for MKMC and $2.0 \mathrm{~ms}$ for FEM. Mean $\mathrm{APD}_{90}$ decreased in association with the infarct scar showing no electrical activity. The decrease was $3.2 \mathrm{~ms}$ for MKMC and $2.2 \mathrm{~ms}$ for FEM.

\section{Discussion}

This work proposes a novel approach for in silico cardiac electrophysiology simulations. The Moving Kriging Mixed Collocation (MKMC) method is implemented to solve the monodomain model on an immersed grid representation of a swine biventricular model. The MKMC method is shown to generate results in very good agreement with FEM, both at baseline and under myocardial infarction conditions. The effects of the presence of an infarct scar on mean LAT and $\mathrm{APD}_{90}$ rendered by the two methods are very similar, with differences below $1 \mathrm{~ms}$.

An infarct border zone is not considered in the myocardial infarction biventricular model. In future studies, the electrophysiological properties and presence of fibrosis in the border zone will be investigated using the immersed grid MKMC method. Also, despite the stimulation protocol applied to the biventricular model resulted in realistic 


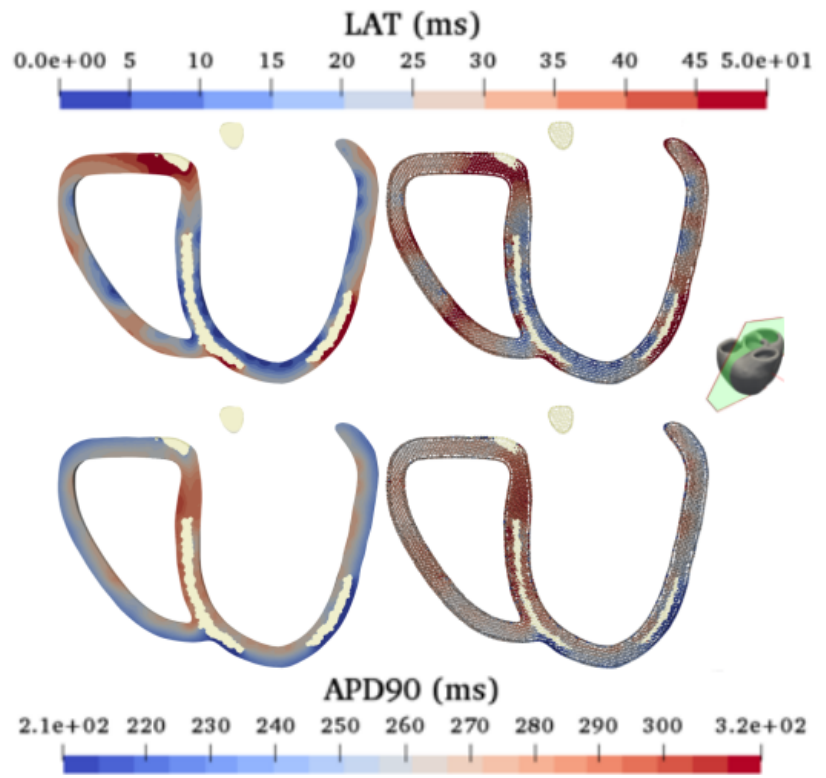

Figure 4. Local activation time (top) and APD duration at $90 \%$ repolarization (bottom) for FEM (left) and MKMC (right) simulations under myocardial infarction conditions.

activation time patterns, further studies including AP propagation in the Purkinje network, under physiological and pathological conditions, are planned to be conducted.

The immersed grid MKMC method is deemed as a very important development for cardiac electrophysiology modeling. The very good agreement with FEM, the fact of not requiring a mesh, and the simplicity to map image data to the immersed grid points altogether allow to construct and solve computational models of arbitrary complexity.

\section{Conclusions}

We demonstrated MKMC method's capability for in silico cardiac electrophysiological simulations in complex 3D models. The automatic generation of an immersed grid directly from medical image data avoids the mesh requirement of FEM and paves the road for the next-generation of cardiac electrophysiology simulators not only on the research desk but also in the operational room.

\section{Acknowledgments}

This work was supported by ERC-StG 638284 (ERC), PID2019-105674RB-I00 (MICINN), LMP124-18 and T39-20R (Aragón Government cofunded by FEDER 20142020). Computations were performed using ICTS NANBIOSIS (HPC Unit at University of Zaragoza).

\section{References}

[1] Abubakar I, Tillmann T, Banerjee A. Global, regional, and national age-sex specific all-cause and cause-specific mor- tality for 240 causes of death, 1990-2013: a systematic analysis for the global burden of disease study 2013. Lancet 2015;385(9963):117-171.

[2] Nguyen TP, Qu Z, Weiss JN. Cardiac fibrosis and arrhythmogenesis: the road to repair is paved with perils. Journal of molecular and cellular cardiology 2014;70:83-91.

[3] Lopez-Perez A, Sebastian R, Izquierdo M, Ruiz R, Bishop M, Ferrero JM. Personalized cardiac computational models: from clinical data to simulation of infarct-related ventricular tachycardia. Frontiers in physiology 2019;10:580.

[4] Lluch E, Doste R, Giffard-Roisin S, This A, Sermesant M, Camara O, De Craene M, Morales HG. Smoothed particle hydrodynamics for electrophysiological modeling: an alternative to finite element methods. In International Conference on Functional Imaging and Modeling of the Heart. Springer, 2017; 333-343.

[5] Mountris KA, Sanchez C, Pueyo E. A novel paradigm for in silico simulation of cardiac electrophysiology through the mixed collocation meshless petrov-galerkin method. In 2019 Computing in Cardiology (CinC). IEEE, 2019; 1-4.

[6] Mountris KA, Pueyo E. The radial point interpolation mixed collocation (rpimc) method for the solution of transient diffusion problems. arXiv200101027 2020;.

[7] $\mathrm{Gu}$ L. Moving kriging interpolation and element-free galerkin method. International journal for numerical methods in engineering 2003;56(1):1-11.

[8] Qu Z, Garfinkel A. An advanced algorithm for solving partial differential equation in cardiac conduction. IEEE Transactions on Biomedical Engineering 1999;46(9):1166-1168.

[9] Rigol M, Solanes N, Fernandez-Armenta J, Silva E, Doltra A, Duchateau N, Barcelo A, Gabrielli L, Bijnens B, Berruezo A, et al. Development of a swine model of left bundle branch block for experimental studies of cardiac resynchronization therapy. Journal of cardiovascular translational research 2013;6(4):616-622.

[10] Doste R, Soto-Iglesias D, Bernardino G, Alcaine A, Sebastian R, Giffard-Roisin S, Sermesant M, Berruezo A, Sanchez-Quintana D, Camara O. A rule-based method to model myocardial fiber orientation in cardiac biventricular geometries with outflow tracts. International journal for numerical methods in biomedical engineering 2019; 35(4):e3185.

[11] Costabal FS, Hurtado DE, Kuhl E. Generating purkinje networks in the human heart. Journal of biomechanics 2016; 49(12):2455-2465.

[12] Durrer D, Van Dam RT, Freud G, Janse M, Meijler F, Arzbaecher R. Total excitation of the isolated human heart. Circulation 1970;41(6):899-912.

Address for correspondence:

Konstantinos A Mountris

University of Zaragoza, Campus Río Ebro, I+D Building, D5.01.1B, Mariano Esquillor, s/n street, 50018, Zaragoza, Spain kmountris@unizar.es 